\title{
Preventing progression to first-episode psychosis in early initial prodromal states
}

\author{
A. Bechdolf, M. Wagner, S. Ruhrmann, S. Harrigan, V. Putzfeld, R. Pukrop, A. Brockhaus-Dumke, \\ J. Berning, B. Janssen, P. Decker, R. Bottlender, K. Maurer, H.-J. Möller, W. Gaebel, H. Häfner, \\ W. Maier and J. Klosterkötter
}

\section{Background}

Young people with self-experienced cognitive thought and perception deficits (basic symptoms) may present with an early initial prodromal state (EIPS) of psychosis in which most of the disability and neurobiological deficits of schizophrenia have not yet occurred.

\begin{abstract}
Aims
To investigate the effects of an integrated psychological intervention (IPI), combining individual cognitive-behavioural therapy, group skills training, cognitive remediation and multifamily psychoeducation, on the prevention of psychosis in the EIPS.
\end{abstract}

\section{Method}

A randomised controlled, multicentre, parallel group trial of 12 months of IPI $v$. supportive counselling (trial registration number: NCT00204087). Primary outcome was progression to psychosis at 12- and 24-month follow-up.

\section{Results}

A total of 128 help-seeking out-patients in an EIPS were randomised. Integrated psychological intervention was superior to supportive counselling in preventing progression to psychosis at 12 -month follow-up $(3.2 \% v$. $16.9 \% ; P=0.008)$ and at 24-month follow-up (6.3\% v. 20.0\%; $P=0.019)$.

\section{Conclusions}

Integrated psychological intervention appears effective in delaying the onset of psychosis over a 24-month time period in people in an EIPS.

\section{Declaration of interest}

None.
Early detection and intervention strategies have led to substantial improvement in the prognosis of a number of non-psychiatric medical conditions. ${ }^{1-3}$ The chronicity of schizophrenia provides the primary rationale for adapting these strategies for schizophrenia. In recent years, criteria based on subthreshold levels of psychotic symptoms (ultra-high-risk criteria) have been found to predict psychosis onset within 12 months in $20-30 \%$ of cases. $^{4-6}$ This approach has provided the opportunity of indicated prevention efforts in individuals at imminent risk of schizophrenia in order to reduce or prevent the devastating effects of the disorder.

Five randomised controlled trials (RCTs) in the ultra-high-risk population have been completed so far. They have included evaluations of low-dose risperidone and cognitive-behavioural therapy (CBT) combined, ${ }^{8}$ CBT alone, ${ }^{9,10}$ olanzapine alone ${ }^{11}$ and omega-3 fatty acids. ${ }^{12}$ Although the results of the treatment phase were statistically borderline ${ }^{10,11}$ or significant ${ }^{8,9,12}$ in favour of the respective experimental condition, these effects were not sustained at $12-{ }^{8}, 24-^{11}$ or $36-$ month ${ }^{13,14}$ follow-up. The increased rate of conversion and return of prodromal symptoms to significantly higher levels after removing the specific intervention led some authors to conclude that interventions in the ultra-highrisk population merely delay conversion to psychosis rather than prevent it. ${ }^{11}$

One of the reasons for the limited efficacy of indicated prevention efforts might be that individuals who meet ultra-highrisk criteria already present with symptoms similar to psychotic symptoms. Thus, in the ultra-high-risk population most of the symptoms, disability and neurobiological deficits associated with schizophrenia might already be present ${ }^{15}$. However, certain subtle, self-experienced thought and perception deficits (basic symptoms) have been described in initial prodromal states of schizophrenia, which may precede the onset of subthreshold psychotic symptoms. ${ }^{15,16}$ Furthermore, prospective data of people with basic symptoms $(n=160)^{15}$ indicate that the 12 -month conversion rate to psychosis is lower $(19 \%)$ and the long-term conversion rate higher $(70 \%$ after 5.4 years) than the 12-month transition rate of the ultra-high-risk population $(20-30 \%) .{ }^{4-6}$ Moreover, crosssectional data indicate that levels of psychopathological symptoms, disability, neurophysiological and neuropsychological deficits are lower in patients with basic symptoms than in patients who fulfil ultra-high-risk criteria. ${ }^{17-19}$ Therefore, it has been hypothesised that people with basic symptoms might be in an early initial prodromal state (EIPS) of psychosis in which symptoms, disability and biological deficits are less severe than in the ultra-high-risk population, and that the EIPS population may therefore be more responsive to preventive interventions than people who already fulfil ultra-high-risk criteria. ${ }^{20,21}$

However, to date, no information on the efficacy of interventions in people in the EIPS is available. Therefore, the present RCT study has been undertaken to test the efficacy of a specifically devised integrated psychological intervention (IPI) compared with supportive counselling in individuals in an EIPS. Integrated psychological intervention consists of individual CBT, modified social skills training, cognitive remediation and multifamily psychoeducation. It was chosen as the experimental condition because (a) the applied strategies have been found to be effective in individuals at ultra-high risk and patients with psychosis; ${ }^{8-10,22-24}$ (b) there is no risk of exposing false positives to possible pharmacological side-effects; and (c) it is an established treatment for anxiety, depression and several other syndromes which are regularly present in the pre-psychotic phase. ${ }^{25,26}$ Supportive counselling was designed to provide a minimal level of support for individuals who were seeking help and clearly in need of support as a result of psychiatric symptoms or concerns relating to functional domains.

This paper presents the 12-month (post-treatment) and 24-month follow-up results of the RCT. The primary outcome 
measure was progression to psychosis (incidences of subthreshold psychosis, first-episode psychosis and first-episode schizophrenia) at 12-month and 24-month follow-up. It was hypothesised that IPI would significantly reduce progression to psychosis compared with supportive counselling.

\section{Method}

This was a multicentre, prospective, randomised trial with two parallel groups assigned to alternative out-patient interventions. Randomisation was achieved by using computer-generated block randomisation stratified by centre. Both interventions were delivered over a 12-month period. Follow-up of participants was conducted for up to 24 months after intake. The protocol was approved by the respective institutional review boards at the Universities of Cologne, Bonn, Dusseldorf and Munich, Germany. All participants provided written informed consent prior to any research activity. This study is registered with Clinicaltrials.gov (registration number NCT00204087).

\section{Setting}

The study took place at four early detection and intervention centres located in the Departments of Psychiatry and Psychotherapy at the Universities of Cologne, Bonn, Dusseldorf and Munich, and was funded within the German Research Network on Schizophrenia. All centres serve as specialised out-patient departments and are designed to provide a low-threshold, nonstigmatising environment. An awareness programme was conducted, which aimed to engage 'at risk' individuals with the early intervention services. Referrals were made from primary healthcare, mental health professionals, counselling services and other youth support services.

\section{Participants}

We used a two-step approach to identifying individuals at risk of psychosis created by Häfner et al. ${ }^{21}$ First, a checklist was provided. For those who met threshold criteria in the checklist, an interview using a specially designed instrument based on the Interview for the Retrospective Assessment of the Onset of Schizophrenia (IRAOS) was performed at the respective centre. This instrument, called the Early Recognition Inventory (ERIraos), ${ }^{27}$ indicated whether the individual at risk fulfilled EIPS or subthreshold psychosis criteria.

\section{Inclusion criteria}

Inclusion criteria were met when patients presented with at least one of ten thought or perceptional basic symptom (Appendix 1 ), which have been found ${ }^{15}$ to predict psychosis in $19 \%$ of cases within 12 months and in $70 \%$ of cases within 5.4 years. A decrease in functioning in conjunction with presentation of a genetic risk of psychosis or a history of obstetric complications has been found to increase the risk of developing psychosis substantially. ${ }^{4-6,28,29}$ For more details on the EIPS criteria see Bechdolf et $a l^{20}$ and Häfner et al. ${ }^{21}$

\section{Exclusion and exit criteria}

In accordance with the concept that the EIPS precedes subthreshold psychotic symptoms (attenuated or brief intermittent psychotic symptoms), the development of subthreshold psychotic symptoms or psychosis served as exit criteria for the trial (see Appendix DS1 for full exclusion and exit criteria).

\section{Treatments}

The same research therapists delivered IPI and supportive counselling, except in Cologne, where 7 patients assigned to supportive counselling were treated by 2 additional psychiatrists, and in Munich, where supportive counselling was delivered to 11 patients by a clinical psychologist and a psychiatrist who were not involved in IPI. No formal measures of adherence to the manual or of therapists competence were employed in the IPI or supportive counselling conditions.

The therapists delivering IPI were CBT-trained clinical psychologists and psychiatrists with at least 2 years of experience in CBT delivery. At the start of the study the therapists were trained in IPI in a comprehensive workshop lasting for several days, and at least once yearly throughout the study. At each site, therapists received expert and/or peer supervision at least once every 2 weeks.

\section{Integrated psychological intervention (IPI)}

Model. The stress-vulnerability model of schizophrenia ${ }^{30}$ and the basic symptom concept ${ }^{15,16}$ serve as the framework of IPI. Thus, improving coping resources and stress management are underlying strategies of the intervention. Given empirical evidence that cognitive thought and perception disorders may precede negative affective states, social withdrawal and decline, ${ }^{15,16}$ a specific cognitive model of the EIPS $^{31}$ was developed, as an extension of the stress-vulnerability model based on recent cognitive models of psychosis. ${ }^{32-34}$ In this model, biological, psychological, social stress and vulnerability factors are presumed to interact to render the person at high risk for the subsequent development of prodromal symptoms. The prodromal symptoms become manifest on exposure to a range of additional stressors, which again may be social, psychological or biological. The occurrence of self-experienced cognitive thought and perception deficits (basic symptoms) could then serve as triggering events for the appraisal of negative beliefs and assumptions. Self-experience of basic symptoms may result in emotional disturbances, such as depression or anxiety, social withdrawal and decline, which jointly contribute to the development and maintenance of symptoms and distress.

Treatment components. The interventions draw on established strategies for first-episode or recurrent schizophrenia, anxiety and depressive disorders. ${ }^{22-26}$

Individual cognitive-behavioural therapy (CBT). Individual CBT was at the core of IPI. Based on our integrative cognitive model ${ }^{31}$ the individual CBT followed the basic principles of cognitive therapy described by Beck $^{35}$ as being formulation driven, structured, based on shared problems and goals, educational, utilising guided discovery as the engine for change, involving homework and being time limited. Depending on the problems presented and the case formulation, therapists adapted the modules described in a manual ${ }^{36}$ (see Table 1 for modules and strategies applied).

Skills training. Scheduling and monitoring of mastery and pleasure activities, 'keeping well' strategies, social perception and social skills training and training in problem-solving were offered in a group format. Each therapy session followed a detailed protocol which outlined the aims of the session, examples of interventions and model responses for the therapist.

Cognitive remediation. Cognitive remediation was offered to address thought and perception deficits (basic symptoms) directly. 
Table 1 Integrated psychological intervention for patients in an early initial prodromal state of psychosis

\begin{tabular}{|c|c|}
\hline Module & Topics \\
\hline $\begin{array}{l}\text { Individual cognitive-behavioural therapy } \\
25 \text { sessions }\end{array}$ & $\begin{array}{l}\text { Assessment and engagement } \\
\text { Psychoeducation } \\
\text { Stress management } \\
\text { Symptom management } \\
\text { Crisis management }\end{array}$ \\
\hline $\begin{array}{l}\text { Group skills training } \\
15 \text { sessions }\end{array}$ & $\begin{array}{l}\text { Positive mood and enjoying } \\
\text { Training social perception and social skills } \\
\text { Mastering difficult situations }\end{array}$ \\
\hline $\begin{array}{l}\text { Cognitive remediation } \\
12 \text { sessions }\end{array}$ & Computer-based training of concentration, attention, vigilance and memory \\
\hline $\begin{array}{l}\text { Psychoeducational multifamily group } \\
3 \text { sessions }\end{array}$ & Information and counselling of relatives or key persons \\
\hline \multicolumn{2}{|c|}{$\begin{array}{l}\text { Applied cognitive and behavioural strategies in individual and group therapy: formulation; collaborative goal-setting; provision of information and education about stress; basic } \\
\text { (Appendix 1) and negative symptoms, depression, anxiety; stress monitoring, relaxation, distraction techniques; self-monitoring of symptoms, thought monitoring, cognitive } \\
\text { restructuring, positive coping, positive reframing and challenging, goal-setting and time management; coping enhancement techniques, normalising self-experience of } \\
\text { neuropsychological deficits, behavioural strategies such as thought stopping, distraction, activity scheduling; exposure techniques; cognitive restructuring of negative and } \\
\text { self-defeating cognitions; relapse prevention; scheduling and monitoring of mastery and pleasure activities, keeping-well strategies, assertiveness and social skills training; } \\
\text { problem-solving. }\end{array}$} \\
\hline
\end{tabular}

It was computerised and based on cognitive exercises from the COGPACK software (Marker Software, Mannheim, Germany), a multimedia cognitive rehabilitation software designed for use with individuals with compromised brain functioning. In each session, three to six tasks were performed, involving repeated practice of exercises for attention, memory and executive functioning. Patients received performance scores, which were recorded and referred to in order to reinforce performance progress. Task parameters were initially made sufficiently easy for each patient to do well. After session 6, tasks were made more difficult, according to the next level of difficulty (low to medium, or medium to severe).

Psychoeducational multifamily groups. In addition, psychoeducational multifamily groups for family members or key persons were offered. These groups provided information on symptoms, course and treatment of at-risk mental states, as detailed in a manual. ${ }^{17,36}$ These sessions aimed to increase the family's understanding of the EIPS and to reduce stress and interpersonal conflicts.

Procedures and manual. Integrated psychological intervention was initially commenced with individual CBT delivered weekly or every 2 weeks, which was then consecutively supplemented by cognitive remediation and psychoeducation of family members or key persons. Following the assessment and engagement phase, and formulation and goal setting, group skills training was offered to participants, depending on availability of places in the group. While participants were attending the weekly group sessions, the frequency of individual sessions was reduced to crisis intervention, and only maintained in individuals who were clinically judged to have a particularly high risk of developing psychosis or dropping out. During the treatment phase of individual CBT, before or after the group intervention, CBT sessions were planned to be weekly, with a reduction in their frequency during the termination phase towards the end of the overall treatment at 12 months. However, the frequency and duration of sessions were intended to be flexible, depending on arrangements made between patients and therapists, as well as being contingent on the mental state of individual patients.

The IPI was detailed in a manual of 137 pages $^{36,37}$ including an overall treatment model, aims of the treatment components and sessions, problem-specific treatment modules, examples of interventions and model responses for the therapist. Additionally, 80 pages of working material for patients were provided. Further details of IPI and case examples are presented elsewhere. ${ }^{37,38}$ The feasibility of the intervention was tested and confirmed in a pilot sample of 12 patients. ${ }^{39}$

\section{Supportive counselling}

The supportive counselling was designed to provide a minimal level of support for individuals who were seeking help and were clearly in need of support as a result of psychiatric symptoms or concerns relating to functional domains. Basic assessment, basic psychoeducation about the at-risk mental state and counselling in a supportive, warm, genuine, empathic and unstructured style were delivered. Supportive counselling was delivered on a one-toone basis, manual based, regularly supervised and lasted for a maximum of 30 sessions over 12 months.

\section{Assessments}

Face-to-face assessment of the development of the primary outcomes - subthreshold psychotic symptoms, psychosis and schizophrenia - were undertaken at each individual treatment session (maximum of 30 assessments) during the treatment phase and at post-treatment (month 12). During the post-treatment phase, patients were regularly asked about the primary outcomes by telephone and by face-to-face interview during the 24-month follow-up. If the telephone interview indicated a deterioration of symptoms and/or an increased risk of conversion, an additional face-to-face interview was conducted. If patients did not attend assessments, every effort was made to follow them up and to complete assessments with regard to primary outcome variables by means of telephone calls and home assessments. If patients were not available for interviews, key persons and hospital admission data were approached to determine conversion status. Patients were classified as converters or non-converters by an independent consultant psychiatrist or senior clinical psychologist. The masking of these independent consultant psychiatrists or clinical psychologists was not formally measured.

Basic symptom inclusion criteria (Appendix 1) were assessed in a semi-structured interview by a short version of the symptom list of the ERIraos and the IROAS. ${ }^{27}$ Inclusion criteria were operationally defined as fulfilled if one of the ten basic symptoms was rated with a severity of at least 1 (corresponding to a mild 
severity). A basic symptom total score was calculated by summing the scores of the ten basic symptom items defining the EIPS. ${ }^{15}$ Genetic risk of schizophrenia as part of the decreased functioning and risk factor criteria was assessed by the IRAOS. ${ }^{40}$ Obstetric complications were assessed by the respective ERIraos module, which was modelled on the Obstetric Complications, Scale (OCS). ${ }^{41}$

The exit criteria to subthreshold psychosis and psychosis (Appendix DS1) were defined in accordance with definitions used by RCTs in the ultra-high-risk population. ${ }^{8-12}$ The exit criteria to the subthreshold psychosis syndrome 'attenuated psychotic symptoms' were assessed using the symptom list of the ERIraos, and were operationally defined as fulfilled if one of the symptoms was rated as present. The subthreshold psychosis syndrome 'brief limited intermittent psychotic symptoms' and psychosis criteria were assessed by the Positive and Negative Syndrome Scale
(PANSS). ${ }^{42}$ In accordance with previous definitions ${ }^{8,9}$ the presence of brief limited intermittent psychotic symptoms or psychosis were operationally defined by cut-off points on PANSS subscales of 4 or more on hallucinations, 4 or more on delusions and 5 or more on conceptual disorganisation or formal thought disorder. After meeting exit criteria, patients were classified by an independent consultant psychiatrist or senior clinical psychologist into one of three categories: (1) subthreshold psychotic symptoms (see online Appendix DS1 for definitions); (2) DSM-IV psychosis diagnosis: ${ }^{43}$ schizophrenia/schizophreniform, schizoaffective disorder, major depression with psychotic features, bipolar disorder with psychotic features, delusional disorder, brief psychotic disorder, brief psychotic disorder not otherwise specified; or (3) DSM-IV schizophrenia/schizophreniform disorder diagnosis. ${ }^{43}$

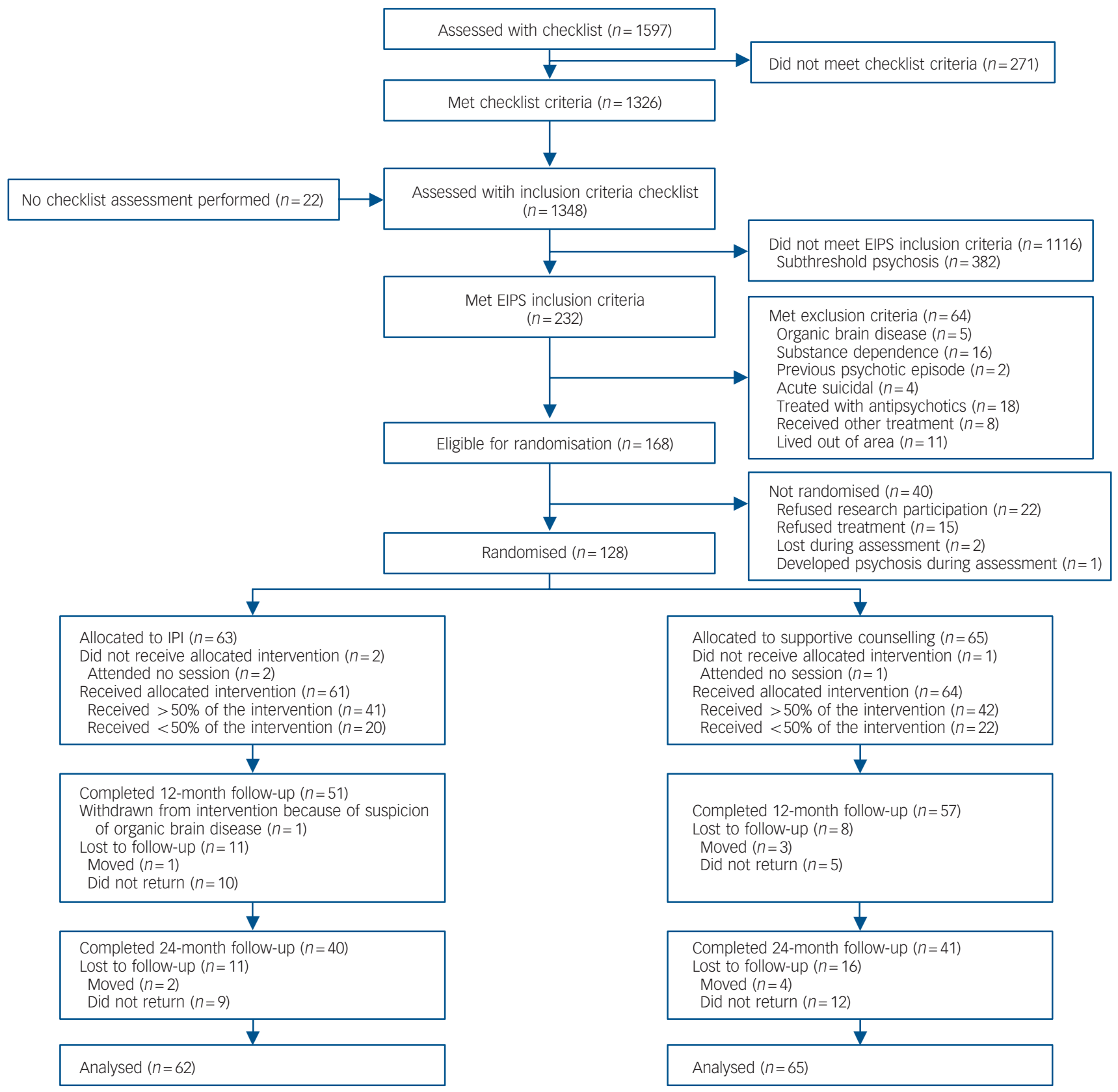

Fig. 1 CONSORT diagram. 
All assessors were experienced clinical psychologists or psychiatrists. They attended a comprehensive workshop lasting several days when the study commenced and yearly throughout the study. Reliability checks of the assessments were performed three times throughout the study with a total number of nine raters. Agreement with a gold standard rating on absence or presence of a symptom from the ERIraos symptom list among eight raters, who were sufficiently trained in the use of the schedule, were good to excellent ${ }^{44}$ (kappa 0.64-0.77). However, one rater, who was still in need of further training, only achieved a kappa of 0.49 (for symptoms present at time of the interview). The reliabilities of the change assessments by the nine raters were good to excellent (kappa 0.63-0.87).

\section{Statistical analysis}

Kaplan-Meier survival analysis assessed differences in time to conversion to subthreshold psychotic symptoms between the two treatment arms over the 24-month follow-up using the logrank test. Estimated survival rates were compared at the 12-month and 24-month points on the survival curve using $z$-tests. Cox regression was applied to assess whether the effects of treatment on survival time remained constant when the impact of the basic symptom total score at baseline was accounted for. These primary survival analyses were performed on all available follow-up data. At 12 -month and 24-month follow-up, a $\chi^{2}$-test was used to calculate the difference in proportions of patients who developed subthreshold psychotic symptoms, psychosis, or schizophreniform/ schizophrenia disorders in the two treatments. The numbers needed to treat (NNT) with CBT to prevent one participant making a conversion were calculated by the reciprocal of the absolute risk reduction. ${ }^{45}$ Two-tailed tests of significance were used in all analyses, with $\alpha$ set to 0.05 .

\section{Results}

\section{Enrolment and participant characteristics}

Recruitment took place from January 2001 to January 2004. Figure 1 illustrates the flow of participant selection. Of 168 eligible individuals, 128 help-seeking out-patients in an EIPS were randomised. Only 15 of the 168 eligible patients (8.9\%) refused to accept the offered interventions, with 25 patients not randomised for other reasons (Fig. 1). Table 2 indicates that the randomised patients were substantially symptomatic and functionally compromised, although patients did not score high on positive psychotic symptoms because these symptoms were among the exclusion criteria. The two treatment groups were reasonably comparable on sociodemographic, symptom and functioning measures at intake (Table 2), with the exception of total basic symptom score, which was slightly higher in the supportive counselling group. Although any differences between the groups are due to chance alone (since the participants were randomised to the treatment groups), it was decided to examine whether the effects of treatment on time to conversion remained constant when the impact of the basic symptom total score at baseline was accounted for.

\section{Adherence to treatment and follow-up}

After randomisation, two patients in the IPI group and one in the supportive counselling group failed to attend any treatment sessions. There were no statistical differences $\left(\chi^{2}=0.003\right.$, $P=0.956)$ in the number of participants who received less than $50 \%$ of the treatment between trial conditions (IPI, $<20$ sessions: $n=22$, 33.8\%; supportive counselling, $<13$ sessions: $n=20$, $31.7 \%$ ). Patients from the supportive counselling group (mean

\begin{tabular}{|c|c|c|}
\hline & $\begin{array}{l}\text { Integrated } \\
\text { psychological } \\
\text { intervention } \\
\quad(n=63)\end{array}$ & $\begin{array}{c}\text { Supportive } \\
\text { counselling } \\
(n=65)\end{array}$ \\
\hline Age, years: mean (s.d.) & $25.2(5.4)$ & $26.8(6.2)$ \\
\hline Male, $n(\%)$ & $39(61.9)$ & $42(64.6)$ \\
\hline Met basic symptoma criteria, $n(\%)$ & 59 (93.7) & 64 (98.5) \\
\hline $\begin{array}{l}\text { Met decreased functioning and risk } \\
\text { factor }^{\mathrm{a}} \text { criteria, } n(\%)\end{array}$ & $14(22.2)$ & $21(32.3)$ \\
\hline $\begin{array}{l}\text { Marital status, } n(\%) \\
\text { Married/cohabiting } \\
\text { Living alone/divorced }\end{array}$ & $\begin{array}{l}28(44.4) \\
35(55.6)\end{array}$ & $\begin{array}{l}24(36.9) \\
41(63.1)\end{array}$ \\
\hline $\begin{array}{l}\text { Employment status, } n \text { (\%) } \\
\text { Full/part time } \\
\text { Student/training } \\
\text { Unemployed/other }\end{array}$ & $\begin{array}{l}11(17.5) \\
34(54.0) \\
18(28.6)\end{array}$ & $\begin{array}{l}16(24.6) \\
27(41.5) \\
22(33.8)\end{array}$ \\
\hline $\begin{array}{l}\text { Housing status, }^{b} n(\%) \\
\text { Independent } \\
\text { Primary family }\end{array}$ & $\begin{array}{l}40(69.0) \\
49(76.6)\end{array}$ & $\begin{array}{l}18(31.0) \\
15(23.4)\end{array}$ \\
\hline $\begin{array}{l}\text { Baseline severity of symptoms } \\
\text { Prodromal symptoms (basic } \\
\text { symptom total score), mean (s.d.) } \\
\text { PANSs subscale score, mean (s.d.) } \\
\text { Positive } \\
\text { Negative } \\
\text { General } \\
\text { MADRS score, mean (s.d.) } \\
\text { Global Assessment of Functioning }\end{array}$ & $\begin{array}{l}9.4(2.9) \\
11.2(4.1) \\
28.2(6.4) \\
19.3(7.8) \\
58.7(11.0)\end{array}$ & $\begin{array}{l}9.2(2.1) \\
11.1(4.1) \\
28.8(6.4) \\
18.9(7.7) \\
60.0(10.1)\end{array}$ \\
\hline \multicolumn{3}{|c|}{$\begin{array}{l}\text { PANSS, Positive and Negative Syndrome Scale; MADRS, Montgomery-Åsberg } \\
\text { Depression Rating Scale. } \\
\text { a. For definitions see Appendix } 1 . \\
\text { b. Six people had other living arrangements. } \\
\text { c. Symptom data are missing for } 0-16 \text { participants. }\end{array}$} \\
\hline
\end{tabular}

number of sessions 15.8 (s.d. $=6.8)$ ) received significantly less treatment $(P<0.001)$ than those in the IPI group $(23.7$ $($ s.d. $=13.1)$ ). After randomisation no patient was withdrawn from the study because of suicidal ideation or worsening of depression.

All 128 randomised patients were included in the primary survival analyses. The median follow-up interval for the entire study period for the 111 participants who did not convert to subthreshold psychosis was 730.0 days (s.d. $=256.6$, range 10 952), while the median interval for the 17 participants who did convert was 237.0 days (s.d. $=188.7$, range $34-664$ ).

\section{Psychosis incidence and time to conversion}

By the end of the treatment phase, 2 of 63 patients in the IPI group and 11 of 65 patients in the supportive counselling group had converted to subthreshold psychosis. During the posttreatment phase, an additional two patients converted in each of the treatment groups. The cumulative conversion rates to subthreshold psychosis at 12 months were $3.2 \%$ for IPI and $16.9 \%$ for supportive counselling (odds ratio $(\mathrm{OR})=6.21,95 \%$ CI $1.32-29.29$ ) and at 24 months $6.3 \%$ for IPI and $20 \%$ for supportive counselling $(\mathrm{OR}=3.69,95 \%$ CI 1.13-12.01). These differences were significant $(P=0.008$ at 12 months and $P=0.019$ at 24 months, as indicated by a $z$-test at each of these points) (Fig. 2). The time to conversion for the entire study period was significantly shorter for the supportive counselling group than the IPI group (IPI: mean 887.1 days, 95\% CI 849-925; supportive counselling: mean 784.2 days, $95 \%$ CI 702-866; log rank: $\left.\chi^{2}=5.43, P=0.020\right)$. The effects of treatment on time to conversion remained statistically significant $(P=0.042)$ when basic symptom total score at baseline was accounted for in a 


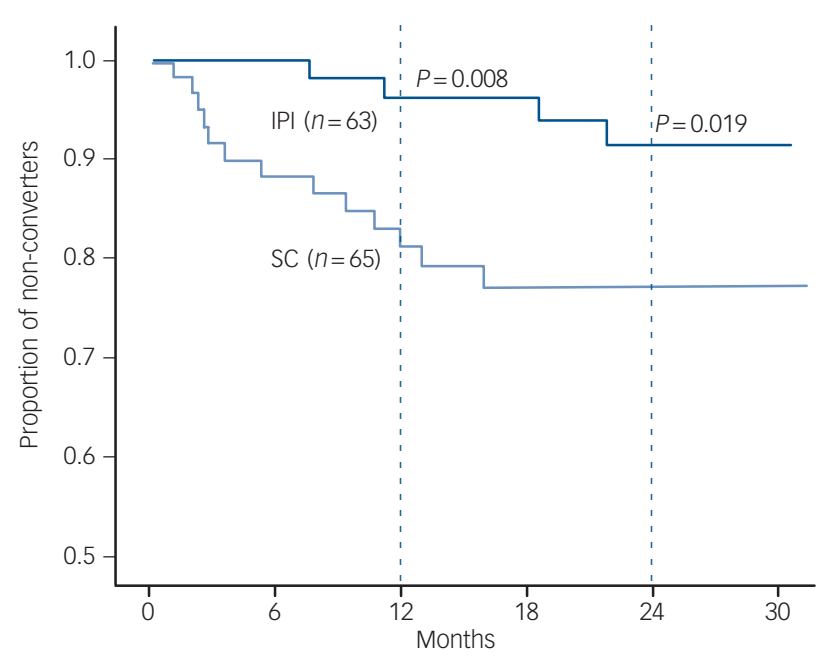

Fig. 2 Kaplan-Meier estimates of the rate of progression to subthreshold psychosis in patients assigned to integrated psychological intervention (IPI, $n=63$ ) or supportive counselling (SC, $n=65)$

Pre-specified $z$-test comparisons for the difference in the proportions converting to subthreshold psychosis at 12 months $(P=0.008)$ and 24 months $(P=0.019)$.

Cox regression model. At 24-month follow-up, significantly fewer patients in the IPI group than in the supportive counselling group had developed psychosis $\left(3.2 \%\right.$ v. $15.4 \% ; \chi^{2}=5.614$, d.f. $=1$, $P=0.018)$ or schizophrenia/ schizophreniform disorder $(1.6 \% v$. $12.3 \% ; P=0.033)$. Details of the participants classified as making conversion are shown in online Table DS1. The NNTs with IPI to prevent one person making the conversion were $8(95 \%$ CI 4.0-8.5) for subthreshold psychotic symptoms, 9 (95\% CI 4.6-42.6) for psychosis and 10 (95\% CI 2.1-19.3) for schizophrenia/schizophreniform disorder. The overall conversion rates did not differ between centres (exact $P=0.785$ ).

\section{Use of antidepressants}

At intake, $17.5 \%(n=11)$ of the IPI group and $20 \%(n=13)$ of the supportive counselling group were being prescribed antidepressants. At 12 months, $9.5 \%$ of the IPI group and $10.8 \%$ of the supportive counselling group and at 24 months, $15.0 \%$ of the IPI group and $9.75 \%$ of the supportive counselling group were being prescribed antidepressant medication. There were no significant differences in antidepressant use between treatment groups.

\section{Discussion}

To our knowledge, this is the first trial to evaluate a specific prevention strategy in individuals putatively in an EIPS. In accordance with our hypothesis, the incidence of and time to conversion to subthreshold psychotic symptoms, psychosis and schizophrenia/schizophreniform disorder during a 12-month treating period was significantly lower for patients who received specially designed IPI than for those who were treated with supportive counselling. This significant difference was maintained throughout the 24-month follow-up.

\section{Comparison with earlier trials in individuals at risk of developing first-episode psychosis}

The present study confirms that EIPS criteria define a clinically symptomatic and functionally compromised population whose risk of developing psychosis is several thousand times higher than the annual risk in the general population. A transition rate of $17 \%$ in the first year during supportive treatment is in line with a $19 \%$ transition rate in the original naturalistic Cologne Early Recognition study. ${ }^{15}$ In accordance with the aim of identifying an especially early stage of the illness, 12 -month transition rates to psychosis in the control condition were lower in our EIPS trial than in RCTs which used ultra-high-risk criteria as inclusion criteria (17\% v. 22-38\%). ${ }^{8-12}$

As regards acceptance of, and adherence to, offered interventions, our trial is reasonably comparable with the psychological intervention trial in an ultra-high-risk population by Morrison et $a l^{9}$ ( $8.9 \%$ v. $2.8 \%$ refused treatment). This contrasts with prevention efforts involving treatment with antipsychotics in ultra-high-risk individuals, which have proven less acceptable to patients (refusal rate of $35.9 \%{ }^{8}$ ) and have relatively low adherence rates $\left(45.2 \%{ }^{8}\right.$ and $\left.54.8 \%{ }^{11}\right)$. The data on unwanted side-effects go along similar lines. There were no reports of side-effects in the psychotherapy studies (Morrison et $a l^{9}$ Addington et $a l^{10}$ or our own study), but McGorry and colleagues $^{8}$ reported some instances of rigour and sedation due to risperidone treatment. In the McGlashan et al trial ${ }^{11}$ weight, pulse rate and fatigue increased significantly (by $8.8 \mathrm{~kg}$ and 9.5 beats/min for weight and pulse rate respectively) in the olanzapine compared with the placebo group. The acute treatment effects of the IPI intervention in comparison with supportive counselling were statistically significant, which was not the case in some trials of ultra-high-risk populations. ${ }^{10,11}$ Moreover, in contrast to the trials in ultra-high-risk populations, ${ }^{8-11,13,14}$ the conversion rates did not increase substantially after removing the specific intervention in the EIPS population. Both findings support the hypothesis that people in an EIPS might be more responsive to treatment than people in later stages of the prodromal phase. $^{20,21}$

\section{Methodological considerations}

First, the overall sample size doubles those of earlier trials ${ }^{8-12}$ and the methodological quality of the trial using the Clinical Trial Assessment Measure ${ }^{46}$ was high, which strengthens the validity of the findings. Second, no formal measures of therapists' adherence to the manual were employed, nor did we use any formal assessments of therapists' competence. However, we believe that the internal validity of the interventions was high, because the framework, setting and supervision differed between IPI and supportive counselling, and additionally, both interventions were detailed in specific manuals. Third, face-to-face contact with therapists within the trial was higher for patients in the IPI group than for patients receiving supportive counselling. Fourth, since all participants received some sort of treatment (IPI or supportive counselling) and there was no 'no treatment' condition, we cannot rule out that participants might have improved without treatment. Finally, since IPI covered a variety of psychological strategies, the trial design did not allow assessment of the relative contribution of the psychological strategies provided.

\section{Clinical consequences}

Despite the limitations mentioned above, the data presented indicate that specifically developed IPI was effective for delaying the onset of subthreshold, first-episode psychosis and schizophrenia over a 24 -month time period. Moreover, the very small numbers of converters after termination of treatment raises the likelihood that interventions in the EIPS indeed prevent psychosis. Integrated psychological therapy was safe, well accepted 
and tolerated by patients, did not produce unpleasant side-effects and might have been helpful in reducing false positives as well. The NNTs of 8 (subthreshold psychotic symptoms) and 9 (psychosis) for IPI to prevent one conversion are clinically meaningful and contrast with for example, NNTs between 71 and 171 (depending on degree of illness) for the treatment of hypertension to prevent one stroke. ${ }^{1}$ Thus, IPI has the potential to improve the prognosis of many young people in an EIPS and could reduce the devastating consequences of schizophrenia for the affected individuals, their families and society.

Andreas Bechdolf, MD, MSC, Department of Psychiatry and Psychotherapy, University of Cologne, Germany, and Department of Psychiatry, University of Melbourne and ORYGEN Research Centre, Melbourne, Australia; Michael Wagner, PhD, Bonn Early Recognition and Intervention Centre for Mental Crises - ZeBB, Section of Experimental and Clinical Psychology, Department of Psychiatry and Psychotherapy, University of Bonn, Germany; Stephan Ruhrmann, MD, Cologne Early Recognition and Intervention Centre for Mental Crises - FETZ, Department of Psychiatry and Psychotherapy, University of Cologne, Germany; Susan Harrigan, MSc, Orygen Research Centre, University of Melbourne, Melbourne, Australia; Verena Putzfeld, Cologne Early Recognition and Intervention Centre for Mental Crises - FETZ, Department of Psychiatry and Psychotherapy, University of Cologne, Germany; Ralf Pukrop, PhD, SCMD, Section of Experimental and Clinical Psychology, Germany, Ralf Pukrop, PhD, SCMD, Section of Experimental and Clinical Psychology, Brockhaus-Dumke, MD, Department of Psychiatry and Psychotherapy, University of Cologne, Germany; Julia Berning, Bonn Early Recognition and Intervention Centre for Mental Crises - ZeBB, Department of Psychiatry and Psychotherapy, University of Bonn, Germany; Birgit Janssen, MD, Dusseldorf Early Recognition and Intervention Centre for Mental Crises - FEZ, Department of Psychiatry and Psychotherapy, University of Dusseldorf, Germany; Petra Decker, BA, Ronald Bottlender, MD, Munich Early Recognition and Intervention Centre for Mental Crises - FETZ Department of Psychiatry and Psychotherapy, University of Munich, Germany; Kurt Maurer, PhD, Schizophrenia Research Unit, Central Institute of Mental Health, Mannheim, Germany; Hans-Jürgen Möller, MD, Department of Psychiatry and Psychotherapy, University of Munich, Germany; Wolfgang Gaebel, MD, Department of Psychiatry and Psychotherapy, University of Dusseldorf, Germany; Heinz Häfner, MD, PhD, Schizophrenia Research Unit, Central Institute of Mental Health, Mannheim, Germany; Wolfgang Maier, MD, Department of Psychiatry and Psychotherapy, University of Bonn, Germany; Joachim Klosterkötter, MD, Department of Psychiatry and Psychotherapy, University of Cologne, Germany

Correspondence: Andreas Bechdolf, MD, MSC, Associate Professor, Department of Psychiatry and Psychotherapy, University of Cologne, Kerpener Str. 62, 50924 Cologne, Germany. Email: andreas.bechdolf@uk-koeln.de

First received 21 Mar 2009, final revision 9 May 2011, accepted 16 Jun 2011

\section{Funding}

This study is part of the German Research Network on Schizophrenia funded by the German Federal Ministry for Education and Research (BMBF, grant $01 \mathrm{Gl}$ 9935). The sponsor of the study had no role in study design, data collection, data analysis, data interpretation, or writing of the report.

\section{Appendix 1}

\section{Inclusion criteria (early initial prodromal state)}

Self-experienced thought and perception deficits (basic symptoms): one or more of the following basic symptoms in the past 3 months several times a week:

(a) thought interferences

(b) thought perseveration

(c) thought pressure

(d) thought blockages

(e) disturbances of receptive language, either heard or read

( $f$ decreased ability to discriminate between ideas and perception, fantasy and true memories

(g) unstable ideas of reference (subject centrism)

(h) derealisation

(i) visual perception disturbances (blurred vision, transitory blindness, partial sight, hypersensitivity to light, etc.)

(j) acoustic perception disturbances (hypersensitivity to sounds or noise, acoasms, etc.)

and/or (k) decrease in Global Assessment of Functioning score of at least 30 points (within the past year) and at least one of the following decreases in functioning and risk factors:

(i) first-degree relative with a lifetime-diagnosis of schizophrenia

(ii) a schizophrenia-spectrum disorder

(iii) pre- or perinatal complications

\section{References}

1 Psaty BM, Lumley T, Furberg CD, Schellenbaum G, Pahor M, Alderman MH et al. Health outcomes associated with various antihypertensive therapies used as first-line agents: a network meta-analysis. JAMA 2003; 289: 2534-44.

2 Peters AL, Davidson MB, Schriger DL, Hasselbald V. A clinical approach for the diagnosis of diabetes mellitus: an analysis using glycosylated hemoglobin levels. Meta-analysis research group on the diagnosis of diabetes using glycosylated hemoglybin levels. JAMA 1996; 276: 1246-52.

3 Adams EK, Breen N, Joski PJ. Impact of the National Breast and Cervical Detection Program on mammography and pap test utilization among white, Hispanic, and African American women: 1996-2000. Cancer 2007; 109: 348-58.

4 Yung AR, Phillips LJ, Yuen HP, McGorry PD. Risk factors for psychosis in an ultra high-risk group: psychopathology and clinical features. Schizophr Res 2004; 67: 131-42.

5 Ruhrmann S, Schultze-Lutter F, Salokangas RK, Heinimaa M, Linszen D, Dingemans $\mathrm{P}$, et al. Prediction of psychosis in adolescents and young adults at high risk: results from the prospective European prediction of psychosis study. Arch Gen Psychiatry 2010; 67: 241-51.

6 Cannon TD, Cadenhead K, Conblatt B, Woods SW, Addington J, Walker E, et al. Prediction of psychosis in youth at high clinical risk: a multi-site longitudinal study in North America. Arch Gen Psychiatry 2008; 65: 28-37.

7 McGorry PD, Yung AR, Phillips L. The 'close-in' or ultra high-risk model: a safe and effective strategy for research and clinical intervention in prepsychotic mental disorder. Schizophr Bull 2003; 29: 771-90.

8 McGorry PD, Yung AR, Phillips L, Yuen HP, Francey S, Cosgrave EM, et al Can first episode psychosis be delayed or prevented? A randomized controlled trial of interventions during the prepsychotic phase of schizophrenia and related psychosis. Arch Gen Psychiatry 2002; 59: 921-8.

9 Morrison AP, French P, Walford L, Lewis SW, Kilcommons A, Green J, et al. Cognitive therapy for the prevention of psychosis in people at ultra-high risk. randomised controlled trial. Br J Psychiatry 2004; 185: 291-7.

10 Addington J, Epstein I, Liu L, French P, Boydell KM, Zipursky RB. A randomized controlled trial of cognitive behavioral therapy for individuals at clinical high risk of psychosis. Schizophr Res 2011; 125: 54-61.

11 McGlashan TH, Zipursky RB, Perkins D, Addington J, Miller T, Woods SW, et al. A randomized, double-blind trial of olanzapine versus placebo in patients prodromally symptomatic for psychosis. Am J Psychiatry 2006 163: 790-9.

12 Amminger GP, Schäfer MR, Papageorgiou K, Klier CM, Cotton SM, Harrigan SM, et al. Long-chain omega-3 fatty acids for indicated prevention of psychotic disorders: a randomized, placebo-controlled trial. Arch Gen Psychiatry 2010; 67: 146-54.

13 Morrison AP, French P, Parker S, Roberts M, Stevens H, Bentall RP, et al. Three year follow-up of a randomised controlled trial of cognitive therapy for the prevention of psychosis in people at ultra-high risk. Schizophr Bull 2007; 33: $682-7$.

14 Phillipps LJ, McGorry PD, Yuen HP, Ward J, Donovan K, Kelly D, et al. Medium term follow-up of a randomized controlled trial of interventions for young people at ultra high risk of psychosis. Shizophr Res 2007; 96: 25-33.

15 Klosterkötter J, Hellmich M, Steinmeyer EM, Schultze-Lutter F. Diagnosing schizophrenia in the initial prodromal phase. Arch Gen Psychiatry 2001; 58: $158-64$.

16 Gross G, Huber G. Psychopathology of basic stages of schizophrenia in view of formal thought disturbances. Psychopathology 1985; 18: 115-25.

17 Bechdolf A, Pukrop R, Köhn D, Tschinkel S, Schultze-Lutter F, Ruhrmann S, et al. Subjective quality of life in subjects at risk for first episode psychosis: a comparison with first episode schizophrenia patients and healthy controls. Schizophr Res 2005; 79: 137-41.

18 Brockhaus-Dumke A, Ruhrmann S, Tendolkar I, Pukrop R, Klosterkötter J. Impaired mismatch negativity (MMN) generation in prodromal and schizophrenia patients. Schizophr Res 2005; 73: 297-310.

19 Pukrop R, Schultze-Lutter F, Ruhrmann S, Brockhaus-Dumke A, Tendolkar I, Bechdolf $A$, et al. Neurocognitive indicators for a transition to psychosis: comparison of subjects at risk who transited to psychosis and who did not transit. Schizophrenia Res 2007; 92: 116-25. 
20 Bechdolf A, Ruhrmann S, Wagner M, Kühn KU, Janssen B, Bottlender R, et al Interventions in the initial prodromal states of psychosis in Germany: concept and recruitment. Br J Psychiatry 2005; 187 (suppl 48): s45-8.

21 Häfner H, Maurer K, Ruhrmann S, Bechdolf A, Klosterkötter J, Wagner M, et al. Are early detection and secondary prevention feasible? Facts and visions. Eur Arch Psychiatry Clin Neurosci 2004; 254: 117-28.

22 Jones C, Cormac I, Silveira da Mota Neto Jl, Campbell C. Cognitive behaviour therapy for schizophrenia. Cochrane Database Syst Rev 2004; 4: CD000524.

23 Pilling S, Bebbington P, Kuipers E, Garety P, Geddes J, Orbach G, et al. Psychological treatment in schizophrenia: I. Meta-analysis of family intervention and cognitive behaviour therapy. Psychol Med 2002; 32: 763-82.

24 Penn DL, Waldheter EJ, Perkins DO, Mueser KT, Lieberman JA. Psychosocial treatment for first-episode psychosis. A research update. Am J Psychiatry 2005; 162: 220-32.

25 Work Group on Panic Disorder, American Psychiatric Association. Practice guideline for the treatment of patients with panic disorder. Am J Psychiatry 1998; 155 (suppl 5): 1-34.

26 American Psychiatric Association. Practice guideline for the treatment of patients with major depressive disorder (revision). Am J Psychiatry 2000; 157 (suppl 4): 1-45.

27 Haefner H, Bechdolf A, Klosterkötter J, Maurer K. Early Detection and Intervention in Psychosis. A Practise Handbook. Schattauer, 2011.

28 Cannon M, Jones PB, Murray RM. Obstetric complications and schizophrenia: historical and meta-analysis. Am J Psychiatry 2002; 159: 1080-92.

29 Geddes JR, Lawrie SM. Obstetric complications and schizophrenia: a metaanalysis. Br J Psychiatry 1995; 167: 786-93.

30 Nuechterlein $\mathrm{KH}$, Dawson ME. A heuristic vulnerability/stress model of schizophrenic episodes. Schizophr Bull 1984; 10: 300-12.

31 Larsen TK, Bechdolf A, Birchwood M. The concept of schizophrenia and phase specific treatment. Psychological treatment in pre-psychosis and nonresponders. J Am Acad Psychoanal Dyn Psychiatry 2003; 31: 209-28.

32 Chadwick PDJ, Birchwood M, Trower P. Cognitive Therapy for Delusions, Voices and Paranoia. John Wiley \& Sons, 1996.

33 Fowler D, Garety P, Kuipers E. Cognitive Behaviour Therapy for People with Psychosis. John Wiley \& Sons, 1995.
34 Kingdon D, Turkington D. Cognitive-Behavioural Therapy for Schizophrenia. Lawrence Erlbaum, 1995.

35 Beck AT. Cognitive Therapy and the Emotional Disorders. International University Press, 1976.

36 Bechdolf A, Puetzfeld V, Gross S, Guettgemanns J. Cognitive Behavioural Therapy in People at Risk of Psychosis. Huber, 2010.

37 Bechdolf A, Veith V, Klosterkötter J. Group intervention for people at high risk of developing psychosis. In Working with People at Ultra-high Risk of Developing Psychosis (eds J Addington, SH Francey, AP Morrison): 169-81. John Wiley \& Sons, 2006.

38 Bechdolf A, Phillips L, Francey S, Leicester S, Morrison AP, Veith V, et al. Recent approaches to psychological interventions for people at risk of psychosis. European Arch Psychiatry Clin Neuroscience 2006: 256: 159-73.

39 Bechdolf A, Veith V, Berning J, Stamm E, Janssen B, Wagner M, et al. Cognitive-behavioural therapy in the pre-psychotic phase. An exploratory study. Psychiatr Res 2005; 136: 251-5.

40 Häfner $H$, Riecher-Rössler A, Hambrecht M, Maurer K, Meissner S, Schmidtke $A$, et al. IRAOS: an instrument for the assessment of onset and early course of schizophrenia. Schizophr Res 1992; 6: 209-23.

41 Lewis SW, Murray RM. Obstetric complications, neurodevelopment deviance and risk of schizophrenia. J Psychiatr Res 1987; 21: 413-21.

42 Kay SR, Opler LA. The Positive and Negative Syndrome Scale (PANSS) for schizophrenia. Schizophr Bull 1987; 13: 507-18.

43 American Psychiatric Association. Diagnostic and Statistical Manual of Mental Disorders (Fourth Edition) (DSM-IV). APA, 1994.

44 Cicchetti DV, Sparrow SA. Developing criteria for establishing interrater reliability of specific items: applications to assessment of adaptive behaviour. Am J Ment Defic 1981; 86: 127-37.

45 Cook RJ, Sackett DL. The number needed to treat: a clinically useful measure of treatment effect. BMJ 1995; 310: 452-4.

46 Tarrier N, Wykes $\mathrm{T}$. Is there evidence that cognitive behaviour therapy is an effective treatment for schizophrenia? A cautious or cautionary tale? Behav Res Ther 2004; 42: 1377-401. 